\title{
Vacuumlike Jet Fragmentation in a Dense QCD Medium
}

\author{
P. Caucal,,$^{1, *}$ E. Iancu, ${ }^{1, \dagger}$ A. H. Mueller, ${ }^{2, \ddagger}$ and G. Soyez ${ }^{1, \S}$ \\ ${ }^{1}$ Institut de Physique Théorique, Université Paris-Saclay, CNRS, CEA, F-91191 Gif-sur-Yvette, France \\ ${ }^{2}$ Department of Physics, Columbia University, New York, New York 10027, USA
}

(Received 2 February 2018; published 6 June 2018)

\begin{abstract}
We study the fragmentation of a jet propagating in a dense quark-gluon plasma. Using a leading, doublelogarithmic approximation in perturbative QCD, we compute for the first time the effects of the medium on multiple vacuumlike emissions. We show that, due to the scatterings off the plasma, the in-medium parton showers differ from the vacuum ones in two crucial aspects: their phase-space is reduced and the first emission outside the medium can violate angular ordering. We compute the jet fragmentation function and find results in qualitative agreement with LHC measurements.
\end{abstract}

DOI: 10.1103/PhysRevLett.120.232001

Introduction.-One of the main objectives of the experimental programs at the RHIC and at the LHC is the characterization of the quark-gluon plasma (QGP) produced in ultrarelativistic heavy ion collisions. An important class of observables used to study this dense form of QCD matter refers to the physics of "jet quenching," i.e., the modifications of the properties of an energetic jet or a hadron due to its interactions with the surrounding medium. A main source for such modifications is the "mediuminduced radiation," responsible, e.g., for the energy lost by the jet at large angles. Within perturbative QCD, this can be computed using the Baier-Dokshitzer-Mueller-PeigneSchiff and Zakharov (BDMPS-Z) formalism [1-3], recently generalized to include multiple medium-induced branchings $[4,5]$. On top of that, "vacuumlike emissions," triggered by parton virtualities, should play an important role in the dynamics of the jets. A complete picture in which the two mechanisms for radiation are simultaneously included from first principles is crucially missing. Existing heuristic scenarios assume, for example, that vacuum parton showers develop first, exactly as in the vacuum, and only then interact with the medium [6,7], or that vacuumlike and medium-induced emissions can be combined with each other, either through postulated mediummodified splitting functions [8-11] or within ad hoc Monte Carlo event generators [12]. A single vacuumlike emission was studied phenomenologically in $[13,14]$.

In this Letter, we study for the first time the effects of the medium on the vacuumlike parton cascades within controlled approximations in perturbative QCD. Our main

Published by the American Physical Society under the terms of the Creative Commons Attribution 4.0 International license. Further distribution of this work must maintain attribution to the author(s) and the published article's title, journal citation, and DOI. Funded by SCOAP . conclusion is that the jet fragmentation can be computed in a leading, double-logarithmic approximation in which such cascades can be factorized from medium-induced emissions. In this approximation, which is common in perturbative QCD [15], jet branching is governed by the usual, DokshitzerGribov-Lipatov-Altarelli-Parisi (DGLAP), splitting functions in the vacuum. However, in the present problem, vacuumlike showers are modified by the medium in two essential ways: a constraint on the allowed phase space and the possibility for additional radiation at large angles. We will show that the jet fragmentation pattern emerging from this picture is in qualitative agreement with the one observed at the LHC.

For simplicity, we consider a "jet" that starts as a colorsinglet quark-antiquark antenna with a small opening angle $\theta_{q \bar{q}} \ll 1$, e.g., produced by the decay of a boosted $W / Z$ boson or a virtual photon. The quark and the antiquark are assumed to have equal energies: $E_{q}=E_{\bar{q}} \equiv E$. We focus on the double-logarithmic approximation (DLA) where gluon emissions are strongly ordered in both energies and emission angles. However, our picture should remain true in the collinear limit in which we relax the ordering in energy. Furthermore, it also applies to the evolution of a quark or gluon jet, provided one replaces $\theta_{q \bar{q}}$ by either the jet radius or the angle of the first emission.

When discussing vacuumlike emissions (VLEs), it is useful to distinguish between three successive stages in the development of a parton cascade: (i) emissions occurring fully inside the medium, (ii) a first emission outside the medium (initiated by a source created inside the medium), and (iii) emissions from sources created outside the medium. This is schematically represented in Fig. 1. The distinction between different types of emissions is controlled by the ratio between the gluon formation time and the "medium size" $L$ (the distance traveled by the jet through the medium). Clearly, the emissions in the third stage follow the same pattern as the genuine parton showers 
$\omega$

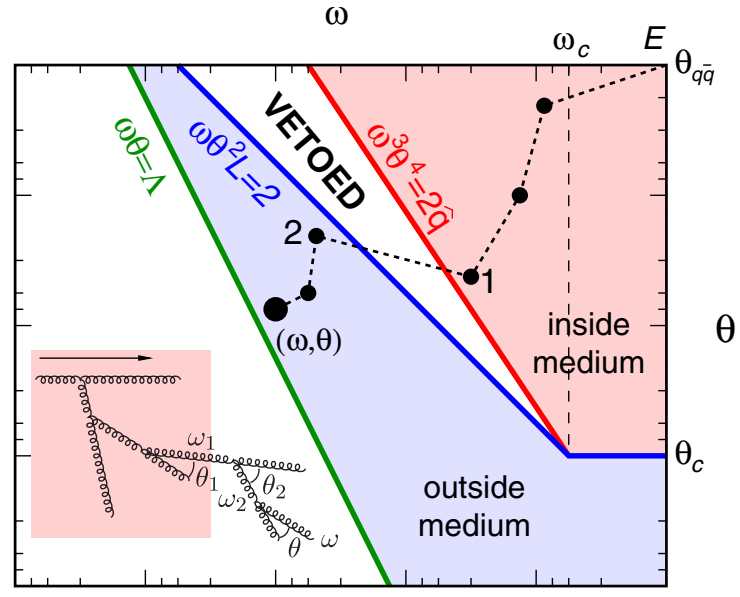

FIG. 1. Schematic representation of the phase space available for VLEs, including an example of a cascade with " 1 " the last emission inside the medium and " 2 " the first emission outside.

in the vacuum. So, in what follows, we shall mainly focus on the first two stages.

Emissions inside the medium.-These are the emissions with formation times smaller than $L$. We start by showing that the VLEs can be factorized from the medium-induced emissions of the BDMPS-Z type (triggered by multiple soft scattering in the medium). This is simply because VLEs proceed fast enough for their formation not to be influenced by the medium.

Formation times.-To understand that, we compare the formation times $t_{f}$ for the vacuumlike and medium-induced emission of a gluon of energy $\omega$ at an angle $\theta . t_{f}$ is determined by the condition that the transverse separation $\Delta r \sim \theta t_{f}$ between the gluon and its parent parton at the time of emission be as large as the gluon transverse wavelength $2 / k_{\perp}$, with $k_{\perp} \simeq \omega \theta$ as its transverse momentum with respect to its emitter. This argument applies to both vacuumlike and medium-induced emissions and implies $t_{f} \simeq 2 \omega / k_{\perp}^{2} \simeq 2 /\left(\omega \theta^{2}\right)$. Then, gluons emitted inside the medium have a minimum $k_{\perp}$ set by the momentum acquired via multiple collisions during formation: $k_{f}^{2} \simeq \hat{q} t_{f}$, with $\hat{q}$ as the jet quenching parameter. This translates into an upper limit $t_{f} \lesssim \sqrt{2 \omega / \hat{q}}$ on the formation time, leaving two possibilities: (a) medium-induced emissions, for which $k_{\perp} \simeq k_{f}$, so the corresponding formation time saturates the upper limit, and (b) VLEs, for which $k_{\perp} \gg k_{f}$, with much shorter formation times. (Emissions with large $k_{\perp} \gg k_{f}$ can also arise from single hard scatterings off the medium. However, such emissions are rare events and by themselves do not give rise to parton cascades.) A VLE is therefore characterized by

$k_{\perp} \gg k_{f} \Leftrightarrow \frac{2}{\omega \theta^{2}} \ll \sqrt{\frac{2 \omega}{\hat{q}}} \Leftrightarrow \omega \gg\left(\frac{2 \hat{q}}{\theta^{4}}\right)^{\frac{1}{3}} \equiv \omega_{0}(\theta)$.
The formation time $t_{f}$ for a medium-induced emission cannot exceed the medium size $L$, meaning that the conditions (1) are only effective for $\omega \leq \omega_{c} \equiv \hat{q} L^{2} / 2$. Emissions with larger energies $\left(\omega \geq \omega_{c}\right)$ behave exactly as in the vacuum: their emission angle can be arbitrarily small and their formation time can be larger than $L$.

The above arguments may seem to imply that all the VLEs with energies $\omega \leq \omega_{c}$ do necessarily have formation times (much) smaller than $L$. However, there are also VLEs that evade the conditions (1) because they are emitted directly outside the medium: $2 /\left(\omega \theta^{2}\right) \gtrsim L$. We discuss such emissions later.

Color (de)coherence.-For emissions by a color-singlet antenna, even a vacuumlike emission obeying (1) could be still affected by the medium, via "color decoherence" [16-19]. In the vacuum, gluon emissions at large angles $\theta \gg \theta_{q \bar{q}}$ are suppressed by the destructive interferences between the quark and the antiquark. This argument can be iterated to conclude that successive emissions in the vacuum are ordered in angles, $\theta_{i+1} \lesssim \theta_{i}$, an ordering which becomes strong $\left(\theta_{i+1} \ll \theta_{i}\right)$ at DLA (see, e.g., [15]). But an antenna propagating through a dense quark-gluon plasma can lose its coherence via rescattering off the medium: the quark and the antiquark suffer independent color rotations, hence the probability that the antenna remains in a color-singlet state decreases with time. The two legs of the antenna start behaving like independent color sources after a time $t \sim t_{\mathrm{coh}}$, where $t_{\mathrm{coh}}$ is the (de)coherence time [18],

$$
t_{\mathrm{coh}}\left(\theta_{q \bar{q}}\right) \equiv\left(\frac{4}{\hat{q} \theta_{q \bar{q}}^{2}}\right)^{1 / 3} .
$$

This timescale becomes comparable to $L$ when $\theta_{q \bar{q}} \sim \theta_{c} \equiv$ $2 / \sqrt{\hat{q} L^{3}}$, with $\theta_{c}$ as the emission angle for the hardest medium-induced emission, with energy $\omega_{c}=\hat{q} L^{2} / 2$.

Antennas with smaller opening angles $\theta_{q \bar{q}} \lesssim \theta_{c}$ cannot lose their coherence; hence, their radiation pattern within the medium is exactly the same in the vacuum. One of our main observations is that antennas with $\theta_{q \bar{q}} \gg \theta_{c}$ (the most relevant case in practice), for which $t_{\text {coh }}\left(\theta_{q \bar{q}}\right) \ll L$ and hence could in principle radiate at large angles $\theta \gg \theta_{q \bar{q}}$, do not do so to the order of interest: they only emit at small angles $\theta \lesssim \theta_{q \bar{q}}$, as for coherent antennas in the vacuum. To see this, note that

$$
\frac{t_{f}}{t_{\mathrm{coh}}}=\frac{\left(2 \hat{q} \theta_{q \bar{q}}^{2}\right)^{1 / 3}}{\omega \theta^{2}}=\frac{\omega_{0}(\theta)}{\omega}\left(\frac{\theta_{q \bar{q}}}{\theta}\right)^{2 / 3}
$$

The loss of color coherence may only affect the emissions at sufficiently large angles, $\theta \gtrsim \theta_{q \bar{q}}$, which overlap with both sources. For VLEs satisfying (1), this implies $t_{f} \ll t_{\text {coh }}$, meaning that the antenna is still coherent at the time of the emission and the would-be large-angle 
emissions are killed by the interference. To summarize, only emissions with $\theta \lesssim \theta_{q \bar{q}}$ are allowed, whether or not they occur at times larger than the decoherence time (2).

Multiple emissions inside the medium.-The leading logarithmic behavior of in-medium parton showers comes from cascades that are strongly ordered in energies and angles, i.e., from cascades with $n$ VLEs satisfying $\theta_{q \bar{q}} \gg$ $\theta_{1} \gg \cdots \gg \theta_{n} \gg \theta_{c}$ and $E \gg \omega_{1} \gg \cdots \gg \omega_{n} \gg \omega_{0}\left(\theta_{n}\right)$. First, note that the formation times $t_{i}=2 /\left(\omega_{i} \theta_{i}^{2}\right)$ are strongly increasing from one emission to the next. This has the important consequence that the cascade is formed in a time $t_{n}$, much smaller then $L$. It also means that the condition (1) is satisfied by all the gluons in the cascade if it is satisfied by the last one. To validate the above picture, we now show that color coherence guarantees the angular ordering and that energy loss can be neglected (at DLA) during the development of the vacuumlike cascade in the medium.

Angular ordering.-For the sake of convenience, color coherence is best discussed in the large $N_{c}$ limit, where the emission of a soft gluon by an antenna can be described as the splitting of the original antenna into two daughter antennas. For any such antenna, say with opening angle $\theta_{i}$, one can apply the same argument about angular ordering as for the original antenna with angle $\theta_{q \bar{q}}$ : VLEs at larger angles $\theta>\theta_{i}$ are strongly suppressed because their formation times are smaller that the decoherence time $t_{\mathrm{coh}}\left(\theta_{i}\right)$ of that antenna.

Energy loss during formation.-We are left with showing that the energy lost via medium-induced radiation remains negligible during the development of a vacuumlike cascade. The hardest medium-induced emission that can occur over the time $t$ has an energy $\omega_{c}(t) \simeq \hat{q} t^{2} / 2$ and a probability of order $\alpha_{s}$ [1,2]. For $t=t_{n} \equiv 2 /\left(\omega_{n} \theta_{n}^{2}\right)$, Eq. (1) implies $\omega_{c}\left(t_{n}\right) \ll \omega_{n}$; i.e., the maximal energy loss is small compared to the energy of the softest gluon in the cascade. The average energy loss, of order $\alpha_{s} \omega_{c}\left(t_{n}\right)$, is even smaller. This argument also shows that, over their formation time, the gluons from the vacuumlike parton showers do not contribute to the energy loss of the jet. However, after being created, they act as additional sources for medium-induced radiation (see below).

First emission outside the medium.-The gluons produced inside the medium are not yet on-shell. Their virtualities are as large as their transverse momenta, themselves bound by the multiple scattering inside the medium: $k_{\perp}^{2} \gg \sqrt{\omega \hat{q}} \gg \Lambda^{2}$, with $\Lambda$ the QCD confinement scale. These partons will thus continue radiating, but their next VLE must occur outside the medium, with a large formation time $2 /\left(\omega \theta^{2}\right) \gg L$, i.e., with an energy $\omega \ll \omega_{L}(\theta) \equiv 2 /\left(L \theta^{2}\right)$. This implies the existence of a gap in the energy of the VLEs, between the lower limit $\omega_{0}(\theta)$ on the last gluon emitted inside the medium, and the upper limit $\omega_{L}(\theta)$ on the first gluon emitted outside the medium. (Interferences between emissions inside and outside the medium are negligible, as their formation times are widely separated.) Since $\omega_{0}(\theta)=\omega_{L}(\theta)=\omega_{c}$ for $\theta=\theta_{c}$, the gap exists only for $\omega<\omega_{c}$, as shown in Fig. 1.

No angular ordering.-Besides the gap in the phase space, the medium has another important effect: the first emission outside the medium can violate angular ordering. (A similar idea was used in [13].) Indeed, all the in-medium sources with $\theta \gg \theta_{c}$ satisfy $t_{\mathrm{coh}}(\theta) \ll L$ and thus lose color coherence after propagating over a distance $L$ in the medium. These sources can then radiate at any angle. On the contrary, the sources with angles smaller than $\theta_{c}$ (hence, $\omega \gtrsim \omega_{c}$; see Fig. 1) are not affected by the medium. They behave as if they were created outside the medium and can radiate only at even smaller angles.

Energy loss after formation.-After being created inside the medium via VLEs, the partons cross the plasma over a distance of order $L$ and hence lose energy via mediuminduced radiation-essentially, as independent color sources. Whereas this is the main mechanism for the energy loss by the jet as a whole, it is less important for the jet fragmentation. Indeed, the typical gluons produced via medium-induced radiation are soft, with $\omega \lesssim \bar{\alpha}_{s}^{2} \omega_{c}$. Via successive democratic branchings [4,5], they transfer their energy to many very soft quanta propagating at large angles $\theta>\theta_{q \bar{q}}$ [20-22]. Hence, such emissions do not matter for the particle distribution inside the jet. (One can show more rigorously that medium-induced emissions do not matter at DLA. However, we believe our physical argument, based on angular separation, to be more insightful.) Furthermore, they do not significantly affect the sources for VLEs: the energy loss is important only for the sources in a small corner of the phase space, at low energies $\omega \lesssim \bar{\alpha}_{s}^{2} \omega_{c}$ and large angles $\theta^{2} \gtrsim\left(1 / \bar{\alpha}_{s}^{3}\right) \theta_{c}^{2}$ [cf. Eq. (1)]. We have checked that the effect of introducing a lower limit $\bar{\alpha}_{s}^{2} \omega_{c}$ on the energies of the VLEs is numerically small. Also, $p_{\perp}$-broadening effects, while important in general, can safely be neglected when computing the gluon multiplicity, since the latter is only sensitive to the angle of emission and not to a change in the direction of the emitter. A complete phenomenological picture should include $p_{\perp}$-broadening and medium-induced emissions. Since such effects go beyond our current level of approximation, we leave them for future work.

Emissions from sources created outside the medium.After a first emission outside the medium, the subsequent emissions follow, of course, the usual pattern of vacuumlike cascades, with angular ordering (and energy ordering in our DLA approximation). The evolution stops when the transverse momentum $k_{\perp} \simeq \omega \theta$ becomes comparable to the hadronization scale $\Lambda$. This implies a lower boundary, $\omega \gtrsim \omega_{\Lambda}(\theta) \equiv \Lambda / \theta$, on the energy of the produced gluons, shown in Fig. 1 together with the other boundaries introduced by the medium. The most interesting region for gluon production - the most sensitive to medium effects highlighted above-is the "outside medium" region at energies $\omega<\omega_{c}$. 
Gluon distribution.-Within the present approximation, it is straightforward to compute the gluon distribution generated by VLEs. To that aim, we compute the double-differential distribution

$$
T(\omega, \theta) \equiv \omega \theta^{2} \frac{d^{2} N}{d \omega d \theta^{2}},
$$

which describes the gluon distribution in both energies and emission angles. Consider a point with coordinates $(\omega, \theta)$ outside the medium. A generic contribution to $T(\omega, \theta)$ can be expressed as the product of a vacuumlike cascade inside the medium, up to an intermediate point $\left(\omega_{1}, \theta_{1}\right)$, followed by a first emission outside the medium, from $\left(\omega_{1}, \theta_{1}\right)$ to $\left(\omega_{2}, \theta_{2}\right)$ and, finally, by a genuine vacuum cascade, from $\left(\omega_{2}, \theta_{2}\right)$ to the measured point $(\omega, \theta)$. This particular contribution yields (at large $N_{c}$ )

$$
\begin{aligned}
T(\omega, \theta)= & \bar{\alpha}_{S} \int_{\theta_{c}^{2}}^{\theta_{q \bar{q}}^{2}} \frac{d \theta_{1}^{2}}{\theta_{1}^{2}} \int_{\omega_{0}\left(\theta_{1}\right)}^{E} \frac{d \omega_{1}}{\omega_{1}} T_{\mathrm{vac}}\left(\omega_{1}, \theta_{1} \mid E, \theta_{q \bar{q}}\right) \\
& \times \int_{\theta^{2}}^{\min \left(\frac{2}{\omega \omega}, \theta_{\bar{q})}^{2}\right)} \frac{d \theta_{2}^{2}}{\theta_{2}^{2}} \\
& \times \int_{\omega}^{\min \left(\omega_{1}, \omega_{L}\left(\theta_{2}\right)\right)} \frac{d \omega_{2}}{\omega_{2}} T_{\mathrm{vac}}\left(\omega, \theta \mid \omega_{2}, \theta_{2}\right),
\end{aligned}
$$

where we have chosen $\theta>\theta_{c}$ for definiteness. The medium effects enter only via the boundaries of the integrations and no ordering is assumed between $\theta_{1}$ and $\theta_{2}$, in agreement with our previous discussion. The explicit factor $\bar{\alpha}_{s} \equiv$ $\alpha_{s} N_{c} / \pi$ refers to the first emission outside the medium and $T_{\text {vac }}\left(\omega_{1}, \theta_{1} \mid E, \theta_{q \bar{q}}\right)$ represents the gluon distribution that would be produced in the vacuum to DLA accuracy, which is well known [15],

$$
\begin{aligned}
T_{\mathrm{vac}}\left(\omega, \theta \mid E, \theta_{q \bar{q}}\right) & =\sum_{n \geq 0} \bar{\alpha}_{s}^{n+1} \frac{1}{(n !)^{2}}\left(\ln \frac{E}{\omega} \ln \frac{\theta_{q \bar{q}}^{2}}{\theta^{2}}\right)^{n} \\
& =\bar{\alpha}_{s} I_{0}\left(2 \sqrt{\bar{\alpha}_{s} \ln \frac{E}{\omega} \ln \frac{\theta_{q \bar{q}}^{2}}{\theta^{2}}}\right),
\end{aligned}
$$

with $I_{0}(x)$ as the modified Bessel function of rank zero. The series expansion in (6) makes explicit the doublelogarithmic enhancement associated with successive emissions simultaneously ordered in energies and angles.

Besides (5), there are also contributions in which either the in-medium cascade or the out-of-medium one is missing. For instance, the first emission by the original antenna can occur directly outside the medium, in which case, there is no cascade inside the medium. Our final results include all these contributions.

The result is shown in Fig. 2 where we plot the ratio $T\left(\omega, \theta^{2}\right) / T_{\text {vac }}\left(\omega, \theta^{2}\right)$ for physically motivated values of the various parameters (specified in the figure). In our DLA approximation, this ratio is 1 for all the points either inside

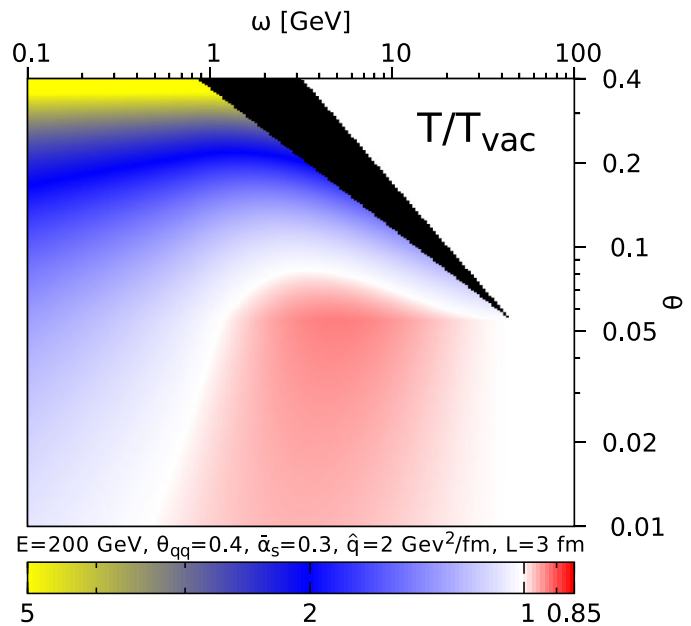

FIG. 2. The ratio $T\left(\omega, \theta^{2}\right) / T_{\text {vac }}\left(\omega, \theta^{2}\right)$ between the two-dimensional gluon distributions in the medium and, respectively, the vacuum, both computed to DLA and for the values of the free parameters $E, \theta_{q \bar{q}}, \bar{\alpha}_{s}, \hat{q}$, and $L$ shown in the figure.

the medium or with $\omega>\omega_{c}$. However, one sees significant deviations from unity for points outside the medium with energies $\omega<\omega_{c}$ : for intermediate values of $\omega$ and relatively small angles $\theta \lesssim 0.1 \theta_{q \bar{q}}$, one sees a small but significant suppression compared to the vacuum (up to $15 \%$ ). For smaller energies and larger angles, $\theta>0.2$, one rather sees a strong enhancement, owing to emissions violating angular ordering.

The fragmentation function.-Finally, we use our above results to compute the jet fragmentation function $D(\omega)$, one of the quantities which is directly measured in the experiments. This is obtained by integrating the doubledifferential distribution over the angles, above the lower limit $\Lambda / \omega$ introduced by hadronization

$$
D(\omega) \equiv \omega \frac{d N}{d \omega}=\int_{\Lambda^{2} / \omega^{2}}^{\theta_{q \bar{q}}^{2}} \frac{d \theta^{2}}{\theta^{2}} T\left(\omega, \theta^{2}\right)
$$

Our results are shown in Fig. 3 for several sets of values for the various parameters. One sees again a slight suppression (relative to vacuum) at intermediate energies, roughly from $2 \mathrm{GeV}$ up to an energy $\sim \omega_{c}$, and a substantial enhancement at lower energies $\omega \lesssim 2 \mathrm{GeV}$. This enhancement is attributed to small-angle emissions inside the medium, radiating at larger angles outside the medium due to the lack of angular ordering. The amount of suppression at intermediate energies grows with both $\hat{q}$ and $L$. This can be easily understood: the logarithmic width of the excluded region $\ln \left(\omega_{0} / \omega_{L}\right)=(1 / 3) \ln \left(\theta^{2} / \theta_{c}^{2}\right)$ rises with $1 / \theta_{c}^{2}=\hat{q} L^{3} / 4$. With increasing $L$, the range in $\omega$ where one sees a enhancement is slowly shrinking, as expected from the phase diagram in Fig. 1.

Remarkably, the above results are in qualitative agreement with the LHC measurements of the fragmentation 


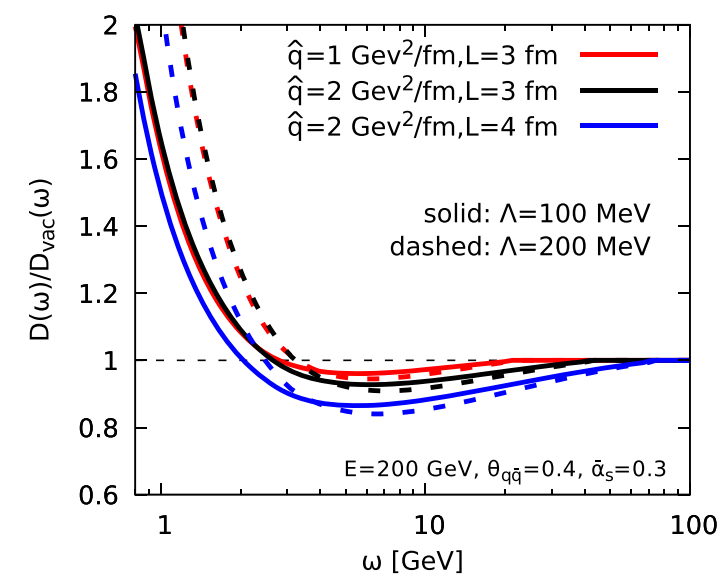

FIG. 3. The ratio $D(\omega) / D_{\text {vac }}(\omega)$ between the fragmentation functions in the medium and, respectively, the vacuum, for different choices for the medium parameters $\hat{q}$ and $L$ and the hadronization scale $\Lambda$ (and fixed values for $E, \theta_{q \bar{q}}$, and $\bar{\alpha}_{s}$ ).

functions for the most central $\mathrm{PbPb}$ collisions [23,24]. Our picture is also coherent with jet shape measurements [25]. Of course, this should be taken with care, in view of our approximations. Yet, it supports the idea that our simple picture, derived in a well-defined limit of perturbative QCD, is close to the actual physical scenario. Our picture can be systematically refined to include, e.g., energy conservation and energy loss, and to compute new observables such as the jet shape. It can also be implemented as a Monte Carlo generator.

We would like to thank Jorge Casalderrey Solana for sharp comments on our manuscript. The work of E. I. and G. S. is supported in part by the Agence Nationale de la Recherche project ANR-16-CE31-0019-01. The work of A. H. M. is supported in part by the U.S. Department of Energy Award No. DE-FG02-92ER40699.

*paul.caucal@ipht.fr

†edmond.iancu@ipht.fr

*amh@phys.columbia.edu

\&regory.soyez@ipht.fr

[1] R. Baier, Y. L. Dokshitzer, A. H. Mueller, S. Peigne, and D. Schiff, Radiative energy loss of high energy quarks and gluons in a finite-volume quark-gluon plasma, Nucl. Phys. B483, 291 (1997).

[2] B. G. Zakharov, Fully quantum treatment of the LandauPomeranchuk-Migdal effect in QED and QCD, JETP Lett. 63, 952 (1996).

[3] U.A. Wiedemann, Gluon radiation off hard quarks in a nuclear environment: Opacity expansion, Nucl. Phys. B588, 303 (2000).

[4] J.-P. Blaizot, F. Dominguez, E. Iancu, and Y. Mehtar-Tani, Medium-induced gluon branching, J. High Energy Phys. 01 (2013) 143.
[5] J.-P. Blaizot, E. Iancu, and Y. Mehtar-Tani, MediumInduced QCD Cascade: Democratic Branching and Wave Turbulence, Phys. Rev. Lett. 111, 052001 (2013).

[6] B. Schenke, C. Gale, and S. Jeon, Martini: An event generator for relativistic heavy-ion collisions, Phys. Rev. C 80, 054913 (2009).

[7] J. Casalderrey-Solana, D. C. Gulhan, J. G. Milhano, D. Pablos, and K. Rajagopal, A hybrid strong/weak coupling approach to jet quenching, J. High Energy Phys. 10 (2014) 019; Erratum, 09 (2015) 175.

[8] X.-N. Wang and X.-f. Guo, Multiple parton scattering in nuclei: Parton energy loss, Nucl. Phys. A696, 788 (2001).

[9] A. Majumder, Hard collinear gluon radiation and multiple scattering in a medium, Phys. Rev. D 85, 014023 (2012).

[10] A. Majumder, Incorporating space-time within mediummodified jet event generators, Phys. Rev. C 88, 014909 (2013).

[11] N. Armesto, L. Cunqueiro, and C. A. Salgado, Q-PYTHIA: A Medium-modified implementation of final state radiation, Eur. Phys. J. C 63, 679 (2009).

[12] K. C. Zapp, F. Krauss, and U. A. Wiedemann, A perturbative framework for jet quenching, J. High Energy Phys. 03 (2013) 080.

[13] Y. Mehtar-Tani and K. Tywoniuk, Jet (de)coherence in $\mathrm{PbPb}$ collisions at the LHC, Phys. Lett. B 744, 284 (2015).

[14] Y. Mehtar-Tani and K. Tywoniuk, Quenching of high- $p_{T}$ jet spectra, arXiv:1707.07361.

[15] Y. L. Dokshitzer, V. A. Khoze, A. H. Mueller, and S. I. Troian, Basics of Perturbative QCD (Frontieres, Gif-surYvette, France, 1991).

[16] Y. Mehtar-Tani, C. A. Salgado, and K. Tywoniuk, Antiangular Ordering of Gluon Radiation in QCD Media, Phys. Rev. Lett. 106, 122002 (2011).

[17] Y. Mehtar-Tani, C. A. Salgado, and K. Tywoniuk, Jets in QCD media: From color coherence to decoherence, Phys. Lett. B 707, 156 (2012).

[18] J. Casalderrey-Solana and E. Iancu, Interference effects in medium-induced gluon radiation, J. High Energy Phys. 08 (2011) 015.

[19] J. Casalderrey-Solana, Y. Mehtar-Tani, C. A. Salgado, and K. Tywoniuk, New picture of jet quenching dictated by color coherence, Phys. Lett. B 725, 357 (2013).

[20] A. Kurkela and U. A. Wiedemann, Picturing perturbative parton cascades in QCD matter, Phys. Lett. B 740, 172 (2015).

[21] J.-P. Blaizot, Y. Mehtar-Tani, and M. A. C. Torres, Angular Structure of the In-Medium QCD Cascade, Phys. Rev. Lett. 114, 222002 (2015).

[22] E. Iancu and B. Wu, Thermalization of mini-jets in a quarkgluon plasma, J. High Energy Phys. 10 (2015) 155.

[23] S. Chatrchyan et al. (CMS Collaboration), Measurement of jet fragmentation in $\mathrm{PbPb}$ and $\mathrm{pp}$ collisions at $\sqrt{s_{N N}}=$ 2.76 TeV, Phys. Rev. C 90, 024908 (2014).

[24] G. Aad et al. (ATLAS Collaboration), Measurement of inclusive jet charged-particle fragmentation functions in $\mathrm{Pb}+\mathrm{Pb}$ collisions at $\sqrt{s_{N N}}=2.76 \mathrm{TeV}$ with the ATLAS detector, Phys. Lett. B 739, 320 (2014).

[25] S. Chatrchyan et al. (CMS Collaboration), Modification of jet shapes in $\mathrm{PbPb}$ collisions at $\sqrt{s_{N N}}=2.76 \mathrm{TeV}$, Phys. Lett. B 730, 243 (2014). 